\title{
Unintentional rapid opioid detoxification
}

\author{
M A Quigley, S H Boyce
}

\begin{abstract}
Naltrexone is a long acting opioid antagonist that is used in rehabilitation programmes to maintain heroin abstinence. It is a relatively new drug prescribed by specialists in substance misuse. Its actions and effects may not be familiar to many accident and emergency doctors or even to the people who take it. A case report is described where accidental ingestion of naltrexone results in a medical emergency by precipitating acute withdrawal in a heroin addict.

(Emerg Med f 2001;18:494-495)
\end{abstract}

Keywords: naltrexone; opioids

\section{Case report}

Naltrexone is a pure opioid receptor antagonist that is prescribed to detoxified heroin addicts to prevent relapse. If ingested by people who are opioid dependent it will precipitate immediate withdrawal with potentially life threatening consequences.

We present a case where the chaotic nature of the presentation, an unreliable history and the unpredictability of the drug reaction contributed to delay in making the diagnosis.

A 27 year old injecting drug user presented 30 minutes after her drink had been "spiked" with an unidentified tablet. Fifteen minutes after ingestion she became unwell complaining of abdominal pain, "not feeling right" and "going crazy". She was distraught, restless and agitated, but did settle when spoken too. Oxygen saturations were $96 \%$ on air, blood pressure $116 / 55$, pulse 80 , blood glucose stick testing $5.2 \mathrm{mmol} / 1$, temperature $36.8^{\circ} \mathrm{C}$ and she was not obviously intoxicated. Abdominal and neurological examinations were normal, there was no evidence of psychiatric illness and pupils were reactive size 8 . She did not display excess sweating, goose flesh or tremor. She was given oral diazepam and after observation for over one hour showed no deterioration and she was removed by the police from the department. More than an hour later (3.5 hours from the original ingestion) she returned as a 999 call with further agitation, double incontinence, vomiting and increasing abdominal pain. This time her pulse was 120 and ECG showed sinus tachycardia otherwise no new clinical findings were evident. In the intervening period she had been informed by an unidentified acquaintance that the tablet was for treatment of heroin withdrawal and when prompted she recognised its name as naltrexone. After admission she received monitoring and treatment with antiemetics and oral diazepam. It took over 24 hours for her agitation and physical symptoms to settle and she was discharged on the second day with oral lofexidine. Follow up was arranged with the substance misuse team.

\section{Discussion}

The oral dose of naltrexone is $25 \mathrm{mg}$ increasing to $50 \mathrm{mg}$ per day and it has a prolonged effect of 24 to 72 hours. Patients have to be opioid free for 7-10 days and are given a supervised naloxone challenge before administration. Side effects include nausea, vomiting, anxiety, nervousness, headache, sleep disturbance, diarrhoea, sweating and excess lacrimation, and joint and muscle pain. ${ }^{1}$ The use of naltrexone improves short-term outcomes in opioid detoxification programmes and it may have a place in the treatment of general substance misuse including alcoholism where it not only exerts a physical effect in preventing alcohol reuse but also reduces the psychological craving. ${ }^{2}$ Ultra rapid detoxification uses naltrexone administered to addicts under deep sedation or general anaesthesia.

When a patient currently taking naltrexone requires opioid analgesia a rapidly acting opioid should be carefully titrated under close monitoring and with resuscitation equipment available. Large amounts of opioid may be required and non-receptor effects can occur (facial swelling, itching and erythema). In this situation there is also an increased risk of cardiorespiratory collapse (personal correspondence from DuPont Pharma). Unexpected overdose is recognised in previous naltrexone patients who return to substance misuse and discover a heightened sensitivity to opioid effects.

Treatment of accidental ingestion of naltrexone in opioid dependants is supportive with intravenous fluids, antiemetics, non-opioid analgesia, benzodiazepines and the treatment of any cardiorespiratory compromise (personal correspondence from DuPont Pharma) and Manelli et $a l^{3}$ Symptoms like confusion, somnolence and visual hallucinations appear within five minutes and last up to 48 hours (personal correspondence from DuPont Pharma). There may be significant fluid losses from vomiting and diarrhoea. There is increased sympathetic activity and catecholamine release and the resultant tachycardia can be used to monitor the reversal of opioid dependence. ${ }^{4}$ Opioid administration, including methadone, is fruitless and potentially dangerous. The precise pharmacodynamics are not completely understood and large differences in serum concentrations of the drug have been demonstrated and are thought to reflect variable first pass metabolism. ${ }^{5}$ The nature, severity and duration of accidental withdrawal varies greatly between people and the clinical course is not predictable. ${ }^{3}$ With more ex-addicts 
maintained on naltrexone in the community, there is increasing potential for this drug to be taken by current opioid dependent people and we may see more cases of unintentional "rapid detoxification" presenting to our accident and emergency departments.

Contributors

Michael Quigley identified the case and wrote up the report. Stephen Boyce performed the literature search and contributed to writing the report.

Funding: none.

Conflicts of interest: none.
1 British Medical Association/Royal Pharmaceutical Society Of Great Britain. British National Formulary. London: Of Great Britain. British Nat
BMA/RPSGB, March 2000.

2 Sinclair D. New treatment options for substance abuse from a public health viewpoint. Ann Med 1998;30:406-11.

3 Manelli P, De Risio S, Pozzi G, et al. Serendipitous rapid detoxification from opiates: the importance of time dependant processes. Addiction. 1999;94:589-91.

4 McDonald T, Hoffman WE, Berkowitz R, et al. Heart rate variability and plasma catecholamines in patients during opiod detoxification. F Neurosurg Anaesthesiol 1999;11:1959.

5 Ferrari A, Berlotti M, Dell'Utri A, et al. Serum time course of naltrexone and $\beta$-naltrexol levels during long-term treat20 . 